\title{
Système $P$ - $M$ de dysgénésie des hybrides, polymorphisme génétique et évolution des populations de Drosophila melanogaster
}

\author{
D. ANXOLABÉHERE et G. PERIQUET * \\ Laboratoire de Génétique des Populations, \\ Université Paris VII, tour 42, 2, place Jussieu, F 75005 Paris \\ * Laboratoire d'Ecologie expérimentale (I.B.E.A.S.), \\ Faculté des Sciences, Parc de Grandmont, F 37200 Tours
}

\begin{abstract}
Résumé
De nombreuses séquences d'ADN répété ont été trouvées chez les eucaryotes. Parmi ces séquences, plusieurs sont des éléments mobiles dont la signification évolutive pose actuellement problème. Chez Drosophila melanogaster deux familles d'éléments mobiles ont été mises en évidence par les travaux portant sur la dysgénésie des hybrides. Dans cet article sont présentées les implications pour la génétique des populations de drosophiles, des connaissances acquises sur l'un de ces systèmes, le système $P$ - $M$.
\end{abstract}

Mots-clés : Dysgénésie des hybrides, polymorphisme, évolution, drosophiles.

\author{
Summary \\ P-M system of hybrid dysgenesis, genetic polymorphism and evolution \\ of Drosophila melanogaster populations
}

All eukaryotic genomes show repeated DNA sequences. Many of these repetitive sequences are jumping genes whose biological role is still debated. In Drosophila melanogaster two families involved in the determinism of the hybrid dysgenies syndrome were identified. The impact of present knowledge of the $P-M$ system on Drosophila population genetics is discussed.

Key-words : Hybrid dysgenesis, polymorphism, evolution, Drosophila.

\section{Introduction}

L'apport des dix dernières années dans la connaissance de l'organisation du matériel héréditaire ouvre des perspectives nouvelles pour l'étude du polymorphisme des populations naturelles et pour l'analyse des processus responsables de leur divergence géné- 
tique. La mise en évidence de la variation du degré de redondance de certaines séquences d'ADN moyennement répétés ainsi que de la variation de position de ces séquences (revue par Bukhari, Shapiro \& Adhya, 1977 ; Shapiro \& Cordell, 1982 ; Whitney \& LAMOREUX, 1982) révèlent chez les eucaryotes, un potentiel de variabilité accru par rapport à celui, déjà considérable, détecté par l'analyse électrophorétique des populations naturelles (LewONTIN, 1974 ; Ayala, 1982).

Chez Drosophila melanogaster l'ADN moyennement répété représente environ 17 p. 100 du génome entier (Young, 1979). Cet ADN est composé d'un certain nombre de séquences classées en différentes familles selon la taille des éléments de base. Si la fonction codante de certaines de ces séquences est bien établie (ARN ribosomaux, ARN de transfert), elle n'a pas été découverte pour toutes, bien que l'on sache que certaines sont effectivement transcrites (SPRAdling \& Rubin, 1981). La moitié de l'ADN moyennement répété de $D$. melanogaster est composé d'environ 50 familles de séquences, dispersées dans l'ensemble du génome (Young \& SchWartz, 1981). Pour un certain nombre de ces familles la mobilité des éléments a été montrée et se trouve à l'origine de réarrangements géniques (SPRAdling \& Rubin, 1981; Bingham, Kidwell \& RuBIN, 1982). Elle peut ainsi constituer un générateur de variabilité génétique pour le génome non répété. Parmi ces éléments, les plus étudiés sont ceux du type copia ( copialike elements » tels : $412,297, \operatorname{mdg} 1, \operatorname{mdg} 3 \ldots$.. Ils sont localisés en une trentaine de sites distribués à travers le génome mais il existe une forte variabilité intra et intersouches de drosophiles quant à cette distribution. Ceci a conduit à penser que ces éléments ont un rôle au cours du développement et dans les processus adaptatifs. Ainsi les résultats de Gvozdev et al. (1981) suggèrent que la localisation des éléments $m d g$ et $m d g 3$ a une influence sur la valeur adaptative individuelle et qu'ils peuvent affecter certains caractères quantitatifs plus rapidement que ne le font les arrangements géniques provoqués par recombinaison.

Deux autres familles d'éléments ont été mises en évidence par les travaux portant sur la dysgénésie des hybrides. Ce phénomène se manifeste dans la lignée germinale de l'un des deux hybrides réciproques provenant de croisement entre certaines souches de type différent. Il se caractérise par un ensemble d'anomalies incluant l'induction d'une stérilité thermodépendante, de mutations souvent instables, d'aberrations chromosomiques, de recombinaisons et de ségrégations anormales. Deux systèmes indépendants en sont actuellement connus, différant notamment par leur type de stérilité : mortalité embryonnaire dans le système I-R (revue par BREgliano et al., 1980) et dysgénésie des gonades (stérilité $G D$ ) dans le système $P-M$ (revue par KIDWELl, 1983).

Dans cet article nous présenterons les implications pour la génétique des populations de $D$. melanogaster des connaissances acquises sur le système $P-M$ de dysgénésie des hybrides.

\section{Présentation du système P-M}

\section{A. Composantes génétiques et caractérisation des individus}

Trois types, $P, Q$ et $M$, mutuellement exclusifs, peuvent être distingués par l'observation de leur descendance dans les croisements effectués avec des souches de références en conditions standard, mais à une température de $28-29^{\circ} \mathrm{C}$ (tabl. 1). 
Dans ces conditions une fraction importante de la descendance d'une femelle de type $M$ et d'un mâle de type $P$ présente les anomalies génétiques précédemment décrites (stérilité $G D$, mutations induites, ...), tandis que les descendants issus du croisement réciproque sont normaux ou présentent ces anomalies à des taux très faibles (KIDWELL, Kidwell \& Sved, 1977 ; Engels, 1979). Dans ces mêmes conditions les descendants de tout croisement entre individus de même type ainsi que ceux effectués entre type $P$ et $Q$ ne manifestent pas de dysgénésie. Enfin les descendants de croisements entre femelle de type $M$ par mâle de type $Q$ présentent un ensemble d'anomalies semblable au syndrome de la dysgénésie hybride à l'exception de la stérilité $G D$. Les individus du croisement réciproque sont eux normaux. Le type $Q$ est ainsi généralement considéré comme « un type $P$ défectif » pour la potentialité de stérilité.

Tableau 1

Résultats des croisements effectués à $28,5^{\circ} \mathrm{C}$ entre les différents types du système P-M. Observation of $F 1$ generation, at $28.5^{\circ} \mathrm{C}$, from crosses between the different types in the P-M system.

\begin{tabular}{|c|c|c|c|}
\hline \multirow{2}{*}{ Femelles } & \multicolumn{3}{|c|}{ Mâles } \\
\hline & $\mathbf{M}$ & Q & $P$ \\
\hline $\mathbf{M}$ & F1 normale & $\begin{array}{l}\text { Mutation } \\
\text { Recombinaison }\end{array}$ & $\begin{array}{l}\text { Stérilité GD, } \\
\text { Mutation } \\
\text { Recombinaison }\end{array}$ \\
\hline Q & F1 normale & F1 normale & F1 normale \\
\hline$P$ & F1 normale & F1 normale & F1 normale \\
\hline
\end{tabular}

Les études génétiques ont montré que dans le système $P-M$ la dysgénésie résultait de l'interaction d'éléments multiples et probablement mobiles (facteurs $P$ ), associés aux chromosomes et d'un état extra-chromosomique sensible appelé cytotype $M$ (ENGELs, $1979,1981)$. Les individus dont les cellules germinales sont résistantes à l'action dysgénésique des facteurs $P$ sont définis comme étant de cytotype $P$. Aux trois types d'individus $P, Q$ et $M$ précédemment définis correspondent ainsi les combinaisons formelles suivantes : type $P$ possédant un cytotype $P$ et des facteurs $P$, type $Q$ possédant un cytotype $P$ et des facteurs $P$ défectifs pour l'induction de la stérilité mais actifs pour l'induction des autres anomalies (mutation et recombinaison mâle) et type $M$ possédant un cytotype $M$ et dépourvu de facteur $P$. Cette interprétation est connue sous le terme de l'hypothèse du facteur $P$ (ENGELs, 1981).

Le tableau 2 présente les quatre types de croisement généralement effectués pour caractériser les individus. En pratique c'est le taux de stérilité $G D$, mesuré par le pourcentage d'ovaires atrophiés chez 25 à 50 femelles F1 hybrides, qui est le critère utilisé. La présence de taux de stérilité $G D$ (supérieurs à 3 à 10 p. 100 selon les auteurs) parmi les hybrides issus du croisement $\mathbf{A}(\rho \mathbf{M} \times \hat{o}$ en examen) révèle la présence de facteurs $\boldsymbol{P}$ chez les mâles analysés. Réciproquement le croisement $A \star$ ( $q$ en examen $\times \hat{o} P$ ) permet de distinguer les mères à cytotype $M(\mathrm{~F} 1$ dysgénésique $)$ de celles à 
cytotype $P$ (F1 non stérile). En pratique le test d'une population se fait sur un échantillon d'une trentaine d'individus croisés en masse. Le résultat est alors une indication moyenne de l'état de la population sans que la caractérisation individuelle puisse être connue. Outre le croisement témoin, on doit aussi effectuer des croisements intra-souches afin de distinguer les phénomènes de dysgénésie hybride, que l'on ne doit observer que dans les croisements $A$ ou $A \star$, d'autres phénomènes de stérilité thermodépendante par atrophie des gonades (Thierry-Mieg, 1976 ; Périquet, 1979, 1980 a).

\section{TABleau 2}

Utilisation des lignées de référence à $28,5^{\circ} \mathrm{C}$ pour caractériser une souche dans le système P-M.

Strain characterisation in the $\mathrm{P}-\mathrm{M}$ system by mean of reference lines (at $28.5^{\circ} \mathrm{C}$ ).

\begin{tabular}{|c|c|c|}
\hline \multirow{2}{*}{ Femelles } & \multicolumn{2}{|c|}{ Mâles } \\
\hline & $\mathbf{P}$ de référence & Souche à déterminer \\
\hline$M$ de référence & Témoin & Croisement A \\
\hline Souche à déterminer & Croisement $\mathrm{A} \star$ & $\begin{array}{l}\text { Test de stérilité } \\
\text { intra-souche }\end{array}$ \\
\hline
\end{tabular}

\section{B. Analyse des composantes du système P-M}

L'analyse biochimique de mutations induites au locus white par le système $P$ - $M$, a permis de montrer que les facteurs $P$ correspondent à une famille d'éléments mobiles d'ADN, dispersés sur l'ensemble du génome des lignées $P$ (Rubin, Kidwell \& BiNGHAM, 1982). Ces éléments, appelés éléments $P$, insérés au locus white ont une taille variant de 0,5 à $1,4 \mathrm{~Kb}$. Par hybridation moléculaire in situ, Bingham, Kidwell \& RuBin (1982) ont repéré une trentaine de sites d'hybridation dans les lignées $P$ et probablement autant dans les lignées $Q$. Ces éléments sont répartis sur l'ensemble des chromosomes mais leurs localisations varient d'une lignée à l'autre. Aucun site d'hybridation avec la sonde $\boldsymbol{P}$ n'a pu être mis en évidence dans les lignées $M$ étudiées (à l'exception d'une d'entre elles cependant).

La mobilité des éléments $P$ a été montrée par le fait que les mutants white induits présentaient l'insertion d'un élément $P$, tandis que les revertants n'en présentaient pas. Le processus de mutation-reversion était ainsi clairement attribué à un processus d'insertion-excision des éléments $P$.

La composante extrachromosomique est pour l'instant moins bien connue. ENGELS (1979) a montré que les modalités de transmission du cytotype n'étaient pas strictement mendeliennes, mais ne suivaient pas non plus totalement les règles d'une hérédité cytoplasmique. Le cytotype apparaît comme une propriété extrachromosomique généralement transmise ne variatur à travers la lignée femelle pendant plusieurs générations. A long terme cependant le cytotype est fonction de déterminants génétiques nucléaires $(M$ ou $P$ ) pouvant être présents sur les trois grands chromosomes. La relation entre 
ces déterminants et les facteurs $P$ n'a pas encore été clairement établie. Une première hypothèse (ENGELS, 1979) fait appel à un déterminisme double avec d'une part les facteurs $P$ et d'autre part les déterminants cytotypiques. Cependant, la localisation d'un facteur $P$ près du locus purple et l'utilisation de chromosomes déficients pour cette région ont permis de proposer une hypothèse de déterminisme unique. Dans un premier cas l'action pourrait être directe : la présence de facteur $\boldsymbol{P}$ entraînant la formation de cytotype $P$ et leur absence déterminant le cytotype $M$, qui pourrait n'être d'ailleurs que l'absence de cytotype $P$ (PériQuet, Green \& AnXolabérère, 1981). Dans un second cas, l'action peut être indirecte : certains sites de transpositions des éléments $P$ correspondraient aux sites des déterminants cytotypiques. Une insertion à proximité ou à l'intérieur d'un déterminant $M$ induirait le changement vers un cytotype $P$. Inversement l'excision de l'élément $P$ rétablirait l'état cytotypique $M$ (PÉRIQuet \& ANXolABÉHÈRE, 1982). Ces hypothèses sont à rapprocher de celle proposée par KIDWELL (1981) dans laquelle les déterminants du cytotype $P$ seraient également des éléments transposables.

\section{Système $\mathrm{P}-\mathrm{M}$ et système $\mathrm{MR}$}

Parallèlement aux recherches sur le système $P$ - $M$, d'autres auteurs (Hiraizumi, 1971 ; et revues par Green, 1980, et par Woodruff, Slatko \& Thompson, 1983) ont mis en évidence dans diverses populations naturelles de D. melanogaster (EtatsUnis, Royaume-Uni, Yougoslavie, Israël, Japon et Australie) de nombreux chromosomes présentant un ensemble de propriétés génétiques semblables à celles rencontrées dans le système $P-M$. Les chromosomes possédant ces activités sont désignés par le sigle MR, pour Mutation-Recombinaison, et sont très probablement porteurs d'éléments mobiles d'ADN (éléments $M R$ ) auxquels ils doivent leurs propriétés (GREEN, 1977). S'agit-il de deux systèmes indépendants ou au contraire l'activité $M R$ n'est-elle que l'un des aspects de l'activité dysgénésique générale due au système $P-M$ ? En d'autres termes existe-t-il des éléments $M R$ indépendants des éléments $P$ décrits cidessus? Dans tous les cas étudiés, l'activité mutagène a pu être corrélée avec l'induction d'une stérilité GD (ENGels, 1980 ; PÉriQuet, Green \& ANXolabéHère, 1981) ; l'existence de deux types d'éléments nécessiterait alors qu'ils soient en un déséquilibre d'association important. Dans les cas où aucune information sur le type éventuel de stérilité associée aux chromosomes $M R$ n'est disponible, on ne peut écarter l'hypothèse que leur activité mutagène soit également due au système $I-R$ de dysgénésie des hybrides. La notation «MR » peut ainsi être composite et l'on doit attendre des recherches complémentaires, probablement par séquençage des différents éléments, pour préciser la nature commune ou différente de ces systèmes.

\section{Système P-M et populations de D. melanogaster}

\section{A. Populations naturelles et des souches de laboratoire}

Dès les premières études, une dichotomie a été observée entre les souches conservées depuis plus d'une vingtaine d'années en laboratoire et les souches récemment capturées dans la nature. Il apparaissait que les souches de laboratoire étaient essentiellement de type $M$ tandis que les souches de la nature étaient $P$ (KIDwelL, KIDwell 
\& SVED, 1977 ; Kidwell, 1979). Les études plus récentes ont porté d'une part sur l'analyse d'un grand nombre de souches récemment capturées et d'autre part sur la caractérisation de l'état actuel de souches capturées plus ou moins anciennement, et conservées depuis en laboratoire.

L'examen des populations actuelles de $D$. melanogaster dans la nature fait apparaître une diversité plus grande que celle primitivement estimée (figure 1). Aux EtatsUnis la majorité des populations est de type $P$ ou $Q$ tandis que les individus $M$ sont absents ou très rares $(1 \mathrm{p}$. 100). En fait certaines populations $P$ analysées plus en détail (ENGels \& Preston, 1980) se sont révélées polymorphes pour les facteurs $P$ (les taux de stérilité $G D$ étant différents selon les individus de la population) et cette situation semble assez générale pour les Etats-Unis (KIDwElı, 1980). La répartition mondiale est plus variée que celle des populations américaines et les trois types de souches ont été observvés (AnXolabéhìre, Nouaud \& PÉriquet, 1982 a ; Bregliano \& KiDWell, 1983 ; Ohishi, Takanashi \& Ishiwa Chigusa, 1982). Des souches $P$ et $Q$ ont été trouvées essentiellement en Afrique équatoriale et en Australie. En Europe de l'ouest et dans les régions méditerranéennes les populations les plus fréquemment rencontrées sont de type $Q$. Les individus de type $P$ sont rares tandis que ceux de type $M$ sont plus fréquents. La présence de cytotype $M$ a été établie en des régions fort diverses : Portugal, Espagne, France, Algérie, Tunisie, Egypte, Inde, U.R.S.S., Japon et Australie. Ainsi donc semble se dessiner une répartition des populations actuelles de $D$. melanogaster parmi lesquelles les facteurs $P$ polymorphes et très fréquents aux Etats-Unis se rencontreraient plus rarement dans les autres régions du monde.
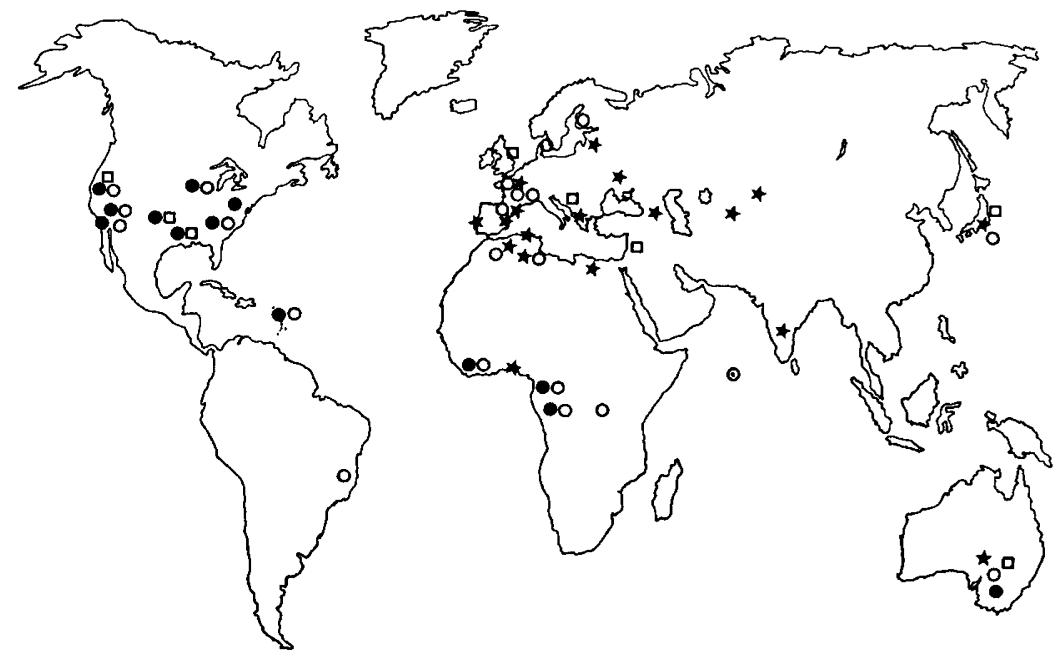

FIG. 1

Distribution des populations actuelles de Drosophila melanogaster pour le système P-M (références dans le texte).

Present distribution of the P-M types in natural populations of Drosophila melanogaster (references in the text).

Type : $\mathrm{P}=\bullet, \mathrm{Q}=0, \mathrm{M}=\star$, chromosome $\mathrm{MR}=\square$.

Unpublished data : $M$ activity of the U.S.S.R. populations (\% of GD sterility in F1 hybrid daughters), Gomel $91 \%$, Uman $87 \%$, Tbilisi $87 \%$, Tachkent $91 \%$, Alma-Ata $94 \%$. 
Au niveau des cytotypes, deux populations $M$, l'une française et l'autre tunisienne, ont été analysées en détail (Anxolabéhère, Nouaud \& Périquet, 1982 b). Dans les deux cas la présence de cytotype $M$ et de cytotype $P$ a pu être mise en évidence révélant ainsi l'existence d'un polymorphisme cytotypique important. Dans ces populations aucun facteur $P$ pouvant déterminer une stérilité $G D$ n'a été trouvé mais une activité mutagène, mesurée par l'induction de mutations aux locus singed et raspberry, a été révélée parmi certains individus de cytotype $P$. Ces résultats suggèrent la présence dans ces populations d'éléments à activité uniquement mutagène.

L'analyse des souches conservées en laboratoire depuis des laps de temps plus ou moins longs, confirme et nuance les premières observations sur l'état de ces souches. Avant la période 1950-1959, la vingtaine de souches étudiées ont toutes été caractérisées comme $M$. C'est dans l'intervalle 1950-1959 que l'on trouve les premières souches qui, mesurées de nos jours, sont de type $P$. Elles sont peu fréquentes (de l'ordre de 10 p. 100), mais on les trouve aussi bien aux Etats-Unis que sur les autres continents. Après cette période, les souches des Etats-Unis sont plus fréquemment de type $P$, puis on trouve une majorité de souches $P$ - $Q$ (de l'ordre de 97 p. 100) dans la période 1970-1979. Dans le reste du monde, les résultats globaux sont semblables mais la fréquence de souches $P-Q$ dans la période 1970-1979 est plus faible (de l'ordre de 70 p. 100) (BREgLiano \& KidWELL, 1983). L'analyse des populations françaises durant la période 1945-1982 apporte quelques informations complémentaires (ANXOLABÉHÈRE, NouAud \& PÉriquet, 1982 a). Les souches antérieures à 1960 sont toutes de type $M$. Durant la période 1960-1976, se rencontrent les trois types de souches $P, Q$ et $M$ mais après cette période aucune souche $P$ n'a pu être mise en évidence, la quasi-totalité des souches étant de type $Q$. L'ensemble de ces résultats ont fait l'objet de plusieurs interprétations et hypothèses que nous allons examiner.

\section{B. Les hypothèses d'interprétation}

Deux hypothèses ont été primitivement proposées. La première, dite «hypothèse de perte aléatoire en élevage» (ENGELS, 1981) suppose que les populations naturelles sont aujourd'hui, comme par le passé, principalement de type $\boldsymbol{P}$ et que les éléments $\boldsymbol{P}$ peuvent être perdus, sous l'effet de la dérive génétique, dans des populations de faible effectif. La mobilité des éléments $P$ expliquerait qu'ils puissent être éliminés d'un chromosome par transposition et excision. Dans des populations d'effectif élevé, cette perte serait extrêmement rare et le phénomène ne pourrait être important que dans les conditions de laboratoire ou dans des populations naturelles isolées. La seconde hypothèse, dite «hypothèse de l'envahissement récent des populations naturelles » (KIDWELL, 1979), propose que la plupart des souches de la nature aient été de type $M$ jusqu'à une période récente. Les facteurs $P$ seraient apparus, ou auraient été activés au sein de ces populations et s'y seraient répandus tout en éliminant les cytotypes $M$. Dans cette hypothèse, les souches anciennes de laboratoire reflètent l'état réel des populations naturelles avant l'installation des éléments $\boldsymbol{P}$ et sont des souches reliques.

Les deux hypothèses présentent une difficulté commune si l'on suppose un double déterminisme avec, d'une part les facteurs $P$, et d'autre part les déterminants chromosomiques du cytotype. Dans chaque hypothèse il faut alors concevoir deux événements successifs pour décrire l'évolution du système $P-M$. Dans la première, la perte des facteurs $P$ chez les souches de type $P$ devrait conduire à des souches $Q$ qui, dans 
un second temps, devraient passer d'un cytotype $P$ à un cytotype $M$, afin de produire les souches $M$ observées en laboratoire. Dans la seconde hypothèse, la difficulté tient de manière réciproque, au processus de passage du cytotype $M$ au cytotype $P$. En effet, dans une première étape, les facteurs $P$, apparus au sein de populations $M$ se seraient trouvés systématiquement associés à des cytotypes $M$. Dans une seconde étape, ces populations à stérilité $G D$ potentiellement élevée, auraient dû évoluer en passant du cytotype $M$ au cytotype $P$, conduisant ainsi aux populations $P$ actuelles. On remarque qu'au contraire le passage du type $M$ au type $P$ (ou inversement) peut être réalisé en une seule étape si l'on suppose que l'apparition (ou la disparition) des éléments $P$ induit directement la transformation d'un cytotype en un autre.

Quelques expériences ont été réalisées dont les résultats peuvent être interprétés dans le cadre de ces deux hypothèses. Kidwell, Novy \& FeEley (1981) ont montré que des populations de laboratoires constituées en proportions diverses d'un mélange initial de types $P$ et $M$, évoluaient toutes vers l'élimination du cytotype $M$ et l'installation du cytotype $P$. Les facteurs $P$ s'installaient également, en conférant à la population un taux d'induction de stérilité $G D$ de l'ordre de 50 p. 100 , ce qui peut être interprété par un état de polymorphisme des facteurs $P$ comparable à celui observé dans les populations naturelles nord-américaines. Lorsque les populations de départ étaient constituées d'un mélange de souches $Q$ et $M$, le cytotype $P$ tendait plus fréquemment à s'installer, mais dans certains cas une proportion importante d'individus de cytotype $M$ était toujours présente à la fin des 25 générations étudiées. Compte tenu des capacités de transposition des éléments $\boldsymbol{P}$, les auteurs suggèrent une évolution par contamination chromosomique et penchent ainsi en faveur de l'hypothèse d'invasion récente des populations naturelles.

Inversement les souches $P$ maintenues en laboratoire en population de faible effectif ont une probabilité de l'ordre de 5 p. 100 par an et par lignée de devenir $M$ (ENGELS in Bingham, Kidwell \& Rubin, 1982). Ce résultat peut être interprété en faveur de l'hypothèse de perte stochastique des éléments, montrant ainsi que les deux hypothèses ne sont pas mutuellement exclusives.

L'étude des populations européennes et plus particulièrement françaises (ANXolABÉHÈre, NouAud \& PÉRIQuet, 1982 a) tout en allant dans le sens de l'hypothèse d'une évolution récente survenue dans la nature, en a cependant suggéré certaines modifications. La répartition actuelle des types $P, Q$ et $M$ pourrait être interprétée comme la progression d'Ouest en Est des facteurs $P$ qui tendraient à s'installer (Bregliano $\&$ KIDWELL, 1983). En fait les premières souches de type $P$ ou $Q$ apparaissent dans les collections entre 1950-1959 aussi bien aux Etats-Unis que dans les autres continents, et la répartition actuelle pourrait simplement traduire des vitesses différentes d'évolution selon les continents, à partir de plusieurs foyers d'origine. D'autre part, l'examen des populations françaises a montré la présence dans les collections de 1965-1975 des types $P$ et $M$, alors que le type $P$ n'a pu être retrouvé après 1976. Au cours de cette période (1965-1975) ces populations semblent donc avoir évolué principalement vers le type $Q$ qui peut ainsi apparaître comme une «stabilisation» des éléments $P$ par perte de la potentialité stérilisante. Dans un tel cas, des individus de type $M$ pourraient être réintroduits par migration ou parce qu'ils sont maintenus à un taux très faible comme dans certaines populations américaines et voir leur fréquence augmenter pour se stabiliser à des niveaux où un polymorphisme cytotypique se maintiendrait, comme dans la population de Nasr'Allah par exemple. Dans cette situation, la réintroduction ultérieure d'éléments $\boldsymbol{P}$ totalement actifs ou leur réactivation (par recombinaison entre 
éléments défectifs par exemple) conduirait ces populations vers une nouvelle phase dysgénésique, en fait créatrice de variabilité génétique. Plutôt qu'une évolution par invasion récente il faut alors envisager un processus cyclique de phase «dysgénésique » suivie de phase stabilisatrice.

Dans cette « hypothèse des phases récurrentes », les éléments $P$ ne sont jamais totalement éliminés mais activés ou inactivés par différents processus et les phases d'envahissements et de stabilisations, non nécessairement synchrones selon les régions, seraient dues à l'action de pressions sélectives opposées. Les pressions défavorables aux éléments $P$ seraient dues, au moins en partie, à leurs effets dysgénésiques en cytotype $M$ et notamment à la stérilité $G D$. Bien que les effets de celle-ci soient minimisés aux températures écologiques de $D$. melanogaster, ils n'en sont pas éliminés pour autant (voir PÉRIQUET, 1979 pour une discussion sur le rôle de la température dans l'évolution de population subissant une stérilité thermo-dépendante). Inversement plusieurs facteurs peuvent favoriser les éléments $P$, le premier étant leur transposabilité. On pourrait également se trouver dans le cas d'un ADN «égoïste 》 (revue par DoolitTle, 1981) si leur vitesse de replication était supérieure à celle du génome de l'hôte. Parmi les facteurs internes on peut faire appel à l'existence de gènes suppresseurs dont l'action compenserait les effets dysgénésiques des éléments. Un tel mécanisme est ainsi suggéré par l'analyse de l'élément copia qui induit la mutation white-apricot $\left(w^{a}\right)$ en s'insérant au locus white. Il existe des gènes suppresseurs de $w^{a}$ (su-wa) dont l'action sur la valeur adaptative d'individus $w^{a}$ a été montrée (PÉRIQUET, $1980 \mathrm{~b}$ ). En cage à population l'élimination des gènes $w^{a}$ est de l'ordre de 10 générations si cet allèle n'est pas associé à un su-wa , tandis qu'elle est de 70 à 80 générations dans le cas contraire, montrant ainsi le rôle d'interaction importante entre mutants et gènes suppresseurs. Parmi les facteurs externes, la résistance à divers insecticides, éventuellement conférée par les éléments $P$, a été recherchée sans que les résultats actuels permettent de conclure définitivement. Les corrélations entre mise en place de la résistance et augmentation de la fréquence de type $P$ pourraient s'expliquer par le fait que les deux phénomènes se soient produits en même temps (Bregliano \& Kidwell, 1983).

On remarquera que les hypothèses «d'envahissement récent » ou de «phases récurrentes» permettent de prévoir des périodes de mutabilité dans les populations naturelles. De telles observations ont été reportées pour les périodes 1937-1945 et 19671975 (GolubovsKY, 1980 ; BERG, 1982) dont l'origine reste évidemment encore à rechercher.

Enfin il faut signaler que chez Drosophila simulans, espèce jumelle de $D$. melanogaster, un phénomène de stérilité semblable à celui produit par le système $P-M$ a été montré (PÉRIQUET, 1981). S'il s'agissait d'un système commun on pourrait alors penser soit à un phénomène d'envahissement semblable dans les deux espèces mais dont le vecteur serait alors externe, soit à une origine ancienne de ces éléments, déjà présents au moment de la formation des deux espèces.

\section{Eléments mobiles, dysgénésie des hybrides et spéciation}

L'observation précédente permet d'introduire les hypothèses faites quant au rôle éventuel des éléments mobiles d'ADN dans l'évolution des espèces. 
Outre le système $P-M$, nous avons précédemment signalé l'existence d'un autre système de dysgénésie des hybrides, le système $I-R$ (Bregliano et al., 1980). Dans ce système la dysgénésie est produite par l'interaction entre des facteurs inducteurs (I) qui sont également des éléments transposables (Picard, 1976, 1979 ; Pelisson, 1981) et un état cellulaire réacteur (R) (Bucheton, 1978, 1979). Comme dans le système $P-M$ il existe une dichotomie, les anciennes souches de laboratoire étant de type $R$ et les souches récemment capturées dans la nature de type $I$. Les mêmes hypothèses ont pu être invoquées pour interpréter l'évolution de ce système, l'apparition des éléments I semblant cependant antérieure (1920 ou avant) à celle des éléments $P$ et plus probablement d'origine américaine (BREgliano \& KidWELL, 1983).

Ces deux systèmes, bien que très similaires, sont cependant distincts (KIDwELL, 1979) et montrent la possibilité de coexistence de plusieurs familles d'éléments mobiles d'ADN pouvant provoquer des phénomènes de dysgénésie des hybrides dont la signification évolutive doit être abordée.

Dans l'hypothèse classique de spéciation par allopatrie (DoBzANSKY, 1970; MaYR, 1970), la création d'une nouvelle espèce débute par l'isolement géographique d'une population. La réduction ou l'absence de flux génique entre l'isolat et la population mère peut être suivie de changements génétiques indépendants, conduisant à la mise en place d'une barrière génétique. Si l'isolement reproductif apparu entre ces deux populations est suffisant pour empêcher tout échange génique en cas de retour à la sympatrie, le processus a conduit à la formation d'une nouvelle espèce.

Compte tenu du phénomène de dysgénésie des hybrides, Bingham, Kidwell \& RuBiN (1982) ont proposé un modèle de spéciation allopatrique faisant appel à l'existence d'éléments mobiles d'ADN, envahissants comme $P$ ou $I$, et présentant des analogies avec la forme provirale des retrovirus (MAJors et al., 1981). Ils proposent qu'il en existe suffisamment de familles pour que des populations séparées durant une certaine période soient contaminées par des types différents et enfin que leurs effets cumulés provoquent la stérilité totale chez les hybrides lorsque ces populations reviennent en sympatrie. Dans ces conditions, la possibilité de divergence entre isolat et population mère pourrait être extrêmement rapide et dans la mesure où ils supposent un délai de trente ans pour l'envahissement par les éléments $P$ et $I$, le processus d'isolement reproductif se déroulerait en un laps de temps considérablement plus court que celui habituellement supposé pour la mise en place par diversification génétique.

Il faut enfin noter que si de tels éléments possèdent des capacités importantes de transposition, on doit s'attendre à ce que ces périodes évolutives s'accompagnent d'une augmentation de la mutabilité des populations. Cette nouvelle variabilité génétique, ponctuelle et par remaniement chromosomique, favorisera l'adaptation de ces populations à leur nouvel environnement, et pourrait même permettre la colonisation de nouvelles niches écologiques, participant ainsi à la fois au processus de l'adaptation des populations et à celui de leur spéciation.

Reçu pour publication le 18 octobre 1982. Accepté pour publication le 2 décembre 1982. 


\section{Remerciements}

Les auteurs remercient M.G. KidWELl et J.C. BrEgliano pour leur avoir communiqué manuscrits sous presse et données non encore publiées. Ce travail a été réalisé au sein de l'E.R.A. 406, du L.A. 340 et du GRECO 44 du C.N.R.S.

\section{Références bibliographiques}

Anxolabehere D., Nouaud D., Periquet G., 1982 a. Etude de la variabilité du système $P-M$ de dysgénésie des hybrides entre populations de Drosophila melanogaster. C.R. Acad. Sc. Paris, 294, 913-918.

Anxolabehere D., Nouaud D., Periquet G., $1982 \mathrm{~b}$. Cytotype polymorphism of the $P$ - $M$ system in two wild populations of Drosophila melanogaster. Proc. Nat. Acad. Sci. U.S.A., 79, 7801-7803.

Ayala F.J., 1982. Biologie moléculaire et évolution. Masson, Paris.

BERG R.L., 1982. High mutability (HM) and hybrid dysgenesis. Genetics, 99, s 4.

Bingham P.M., Kidwell M.G., Rubin G.M., 1982. The molecular basis of $P$-M hybrid dysgenesis : the role of the $P$ element, a P-strain-specific transposon family. Cell., 29, 995-1004.

Bregliano J.C., Kidwell M.G., 1983. Hybrid dysgenesis determinants. In : Shapiro J., Mobile genetic elements. Academic Press, London, in press.

Bregliano J.C., Picard G., Bucheton A. ,Pelisson A., Lavige J.M., L'Heritier P., 1980. Hybrid dysgenesis in Drosophila melanogaster. Science, 207, 606-611.

Bucheton A., 1978. Non mendelian female sterility in Drosophila melanogaster : influence of aging and thermic treatments. Heredity, 41, 357-369.

Bucheton A., 1979. Non mendelian female sterility in Drosophila melanogaster : influence of aging and thermic treatments. III - Cumulative effect induced by these factors. Genetics, 93, 131-142.

Bukhari A.I., Shapiro J.A., AdhYA S.L., 1977. DNA insertion elements, plasmids and episomes. Eds Cold Spring Harbor Lab., New York.

DobZhansKy T. ,1970. Genetics of the evolutionary process. Columbia university press. New York.

Doolitrle W.F., 1981. Selfish DNA after fourteen months. In : Dover G., Flavel R., Genomic evolution, 3-28. Academic Press, London.

ENGELs W.R., 1979. Hybrid dysgenesis in Drosophila melanogaster : rules of inheritance of female sterility. Genet. Res. Camb., 33, 219-236.

ENGELS W.R., 1981. Hybrid dysgenesis and the stochastic loss hypothesis. Cold Spring Harbor Symp. Quant. Biol., 45, 561-565.

ENGELS W.R., 1981. Germline hypermutability in Drosophila and its relation to hybrid dysgenesis and cytotype. Genetics, 98, 565-587.

Engels W.R., Preston C.R., 1980. Components of hybrid dysgenesis in a wild population of Drosophila melanogaster. Genetics, 95, 111-128.

GolubovsKy M.D., 1980. Mutational process and microevolution. Genetica, 52-53, 139-149.

GreEn M.M., 1977. Genetic instability in Drosophila melanogaster : De novo induction of putative insertion mutations. Proc. Nat. Acad. Sci. U.S.A., 74, 3490-3493.

Green M.M., 1980. Transposable elements in Drosophila and other diptera. Ann. Rev. Genet., 14, 109-120. 
Gvozdev V.A., Belyaeva E.S., Ilyin Y.V., Amosova I.S., Kaidanov L.Z., 1981. Selection and transposition of mobile dispersed genes in Drosophila melanogaster. Cold Spring Harbor Symp. Quant. Biol., 45, 673-685.

Hiraizumi Y., 1971. Spontaneous recombination in Drosophila melanogaster. Proc. Nat. Acad. Sci. U.S.A., 68, 268-270.

Kidwell M.G., 1979. Hybrid dysgenesis in Drosophila melanogaster : the relationship between the $P-M$ and $I-R$ interaction systems. Genet. Res. Camb., 33, 205-217.

Kidwell M.G., 1980. The potential of natural populations with respect to the $P$ - $M$ system of hybrid dysgenesis in Drosophila melanogaster. Genetics, 94, s 53 - s 54.

KIDWell M.G., 1981. Hybrid dysgenesis in Drosophila melanogaster : the genetics of cytotype determination in a neutral strain. Genetics, 98, 275-290.

KIDWell M.G., 1983. Intraspecific hybrid sterility. In : Ashburner M., Carson H.L., Thompson J.N. Jr., The genetics and biology of Drosophila, vol. 3 c. Academic Press, London, in press.

Kidwell M.G., Kidwell J.F., Sved J.A., 1977. Hybrid dysgenesis in Drosophila melanogaster : a syndrome of aberrant traits including mutation, sterility and male recombination. Genetics, 86, 813-833.

Kidwell M.G., Novy J.B., Feeley S.M., 1981. Rapid unidirectional change of hybrid dysgenesis potential in Drosophila. J. Hered., 72, 32-38.

LEWONTIN R.C., 1974. The genetic basis of evolutionary change. Columbia University Press, New York.

Majors J.E., Swanstrom R., Delorbe W.J., Payne G.S., Hughes S.H., Ortiz S., QuinTRELl N., BISHOP J.M., VARMUS H.E., 1981. DNA intermediates in the replication of retroviruses are structurally (and perhaps functionally) related to transposable elements. Cold. Spring Harbor Symp. Quant. Biol., 45, 731-738.

Mayr E., 1970. Animal, species and evolution. Harvard University Press, Cambridge (Massachusets).

Ohishi K., Takanashi E., Ishiwa Chigusa S., 1982. Hybrid dysgenesis in natural populations of Drosophila melanogaster in Japan. I. Complete absence of the $P$ factor in an island population. Jpn. J. Genet., 57, 423-428.

Pelisson A., 1981. Contribution à l'étude de la dysgénésie hybride chez Drosophila melanogaster : poursuite de l'analyse génétique du facteur inducteur. Thèse de doctorat d'Etat, Université de Clermont-Ferrand II.

Periquet G., 1979. Maintenance of the «atrophie gonadique » character in Drosophila melanogaster : theoretical models for natural and experimental evolution. Genetica, 51, 33-43.

Periquet G., 1980 a. «Atrophie gonadique» character and hybrid dysgenesis in Drosophila melanogaster. Biol. Cellulaire, 39, 7-12.

Periquet G., 1980 b. Two locus systems in evolution : selection in Drosophila melanogaster at the white-apricot and the suppressor of white-apricot loci. Genetika, 12, 103-110.

Periquet G., 1981. Hybrid sterility in Drosophila simulans : relationships with the hybrid dysgenesis syndrome in Drosophila melanogaster. Heredity, 46, 255-261.

Periquet G., Green M., Anxolabehere D., 1981. Corrélation entre activité mutagène et dysgénésie des gonades dans une souche de Drosophila melanogaster. Biol. Cell., 42, $181-184$.

Periquet G., Anxolabehere D., 1982. Elements causing hybrid dysgenesis on the second chromosome of Drosophila melanogaster. Mol. Gen. Genet., 186, 309-314.

PICARD G., 1976. Non mendelian female sterility in Drosophila melanogaster : hereditary transmission of $I$ factor. Genetics, 83, 107-123.

PiCard G., 1979. Non mendelian female sterility in Drosophila melanogaster : principal characteristics of chromosomes from inducer and reactive origin after chromosomal contamination. Genetics, 91, 455-471. 
Rubin G.M., Kidwell M.G., Bingham P.M., 1982. The molecular basis of $P-M$ hybrid dysgenesis : the nature of induced mutations. Cell., 29, 987-994.

Shapiro J.A., Cordell B., 1982. Eukaryotic mobile and repeated genetic elements. Biol. Cell., 43, 31-54.

Spradling A.C., RUBin G.M., 1981. Drosophila genome organization : conserved and dynamic aspects. Ann. Rev. Genet., 15, 219-264.

THIERRY-MiEg D., 1976. Study of a temperature-sensitive mutant Grandchildlesslike in Drosophila melanogaster. J. Microscopie Biol. Cell., 25, 1-6.

Whitney J.B., LAMOREuX M.L., 1982. Transposable elements controlling genetic instabilities in mammals. J. Hered., 73, 12-18.

WoodrufF R.C., Slatko B.E., ThOMPSON J.N. Jr, 1983. Factors affecting mutation rate in natural populations. In : AshBuRner M., CARSON H.L., ThOMPSON J.N. Jr, The genetics and biology of Drosophila, vol. $3 \mathrm{c}$. Academic Press, London, in press.

Young M.W., 1979. Middle repetitive DNA : a fluid component of the Drosophila genome. Proc. Natl. Acad. Sci. U.S.A., 76, 6274-6278.

Young M.W., Schwartz H.E., 1981. Nomadic genes families in Drosophila. Cold Spring Harbor Symp., 45, 629-640. 